\title{
Sequential Aggregate Signatures with Lazy Verification from Trapdoor Permutations (Extended Abstract)
}

\author{
Kyle Brogle ${ }^{1, \star}$, Sharon Goldberg ${ }^{2}$, and Leonid Reyzin ${ }^{2}$ \\ 1 Stanford University Department of Computer Science \\ Stanford, CA 94305 USA \\ broglek@stanford.edu \\ 2 Boston University Department of Computer Science \\ Boston, MA 02215 USA \\ \{goldbe,reyzin\}@cs.bu.edu
}

\begin{abstract}
Sequential aggregate signature schemes allow $n$ signers, in order, to sign a message each, at a lower total cost than the cost of $n$ individual signatures. We present a sequential aggregate signature scheme based on trapdoor permutations (e.g., RSA). Unlike prior such proposals, our scheme does not require a signer to retrieve the keys of other signers and verify the aggregate-so-far before adding its own signature. Indeed, we do not even require a signer to know the public keys of other signers!

Moreover, for applications that require signers to verify the aggregate anyway, our schemes support lazy verification: a signer can add its own signature to an unverified aggregate and forward it along immediately, postponing verification until load permits or the necessary public keys are obtained. This is especially important for applications where signers must access a large, secure, and current cache of public keys in order to verify messages. The price we pay is that our signature grows slightly with the number of signers.

We report a technical analysis of our scheme (which is provably secure in the random oracle model), a detailed implementation-level specification, and implementation results based on RSA and OpenSSL. To evaluate the performance of our scheme, we focus on the target application of BGPsec (formerly known as Secure BGP), a protocol designed for securing the global Internet routing system. There is a particular need for lazy verification with BGPsec, since it is run on routers that must process signatures extremely quickly, while being able to access tens of thousands of public keys. We compare our scheme to the algorithms currently proposed for use in BGPsec, and find that our signatures are considerably shorter nonaggregate RSA (with the same sign and verify times) and have an order of magnitude faster verification than nonaggregate ECDSA, although ECDSA has shorter signatures when the number of signers is small.
\end{abstract}

* Work done while at Boston University.

X. Wang and K. Sako (Eds.): ASIACRYPT 2012, LNCS 7658, pp. 644-662, 2012.

(C) International Association for Cryptologic Research 2012 


\section{Introduction}

Aggregate signatures schemes allow $n$ signers to produce a digital signature that authenticates $n$ messages, one from each signer. This can be securely accomplished by simply concatenating together $n$ ordinary digital signatures, individually produced by each signer. An aggregate signature is designed to maintain the security of this basic approach, while having length much shorter than $n$ individual signatures. To achieve this, many prior schemes e.g., LMRS04 Nev08] relied on a seemingly innocuous assumption; namely, that each signer needs to verify the aggregate signature so far, before adding its own signature on a new message. In this paper, we argue that this can make existing schemes unviable for many practical applications, (in particular, for BGPsec Lep12 / Secure BGP [KLS00]) and present a new scheme based on trapdoor permutations like RSA that avoids this assumption. In fact, our scheme remains secure even if a signer does not know the public keys of the other signers.

\subsection{Aggregate Signatures from Trapdoor Permutations}

Boneh, Gentry, Lynn, and Shacham BGLS03 introduced the notion of aggregate signatures, in which individual signatures could be combined by any third party into a single constant-length aggregate. The [BGLS03] scheme is based on the bilinear Diffie-Hellman assumption in the random oracle model BR93]. Subsequent schemes [LMRS04 Nev08] were designed for the more standard assumption of trapdoor permutations (e.g., as RSA [RSA78]), but in a more restricted framework where third-party aggregation is not possible. Instead, the signers work sequentially; each signer receives the aggregate-so-far from the previous signer and adds its own signature 1

Lysyanskaya, Micali, Reyzin, and Shacham [LMRS04] constructed the first sequential aggregate signature scheme from trapdoor permutations, with a proof in the random oracle model 2 However, their scheme has two drawbacks: the trapdoor permutation must be certified (when instantiating the trapdoor permutation with RSA, this means that each signer must either prove certain properties of the secret key or else use a long RSA verification exponent), and each signer needs to verify the aggregate-so-far before adding its own signature. Neven [Nev08] improved on [LMRS04] by removing the need for certified trapdoor permutations, but the need to verify before signing remained. Indeed, a signer who adds its own signature to an unverified aggregate in both LMRS04 and [Nev08] (or, indeed, in any scheme that follows the same design paradigm) is exposed to a devastating attack: an adversary can issue a single malformed

${ }^{1}$ The need for the random oracle model was removed by Lu, Ostrovsky, Sahai, Shacham, and Waters LOS+06, who constructed sequential aggregate signatures from the bilinear Diffie-Hellman assumption; however, it is argued in CHKM10. that this improvement in security comes at a considerable efficiency cost. See also RS09 CSC09 for other proposals based on less common assumptions.

${ }^{2}$ Bellare, Namprempre, and Neven [BNN07] showed how the schemes of BGLS03] and LMRS04] can be improved through better proofs and slight modifications. 
aggregate to the signer, and use the signature on that malformed message to generate a valid signature on a message that the signer never intended to sign (we describe the attack in the full version of the paper BGR11b).

The nonsequential scheme of BGLS03 does not, of course, require verification before signing. The only known sequential aggregate scheme to not require verification before signing is the history-free construction of Fischlin, Lehmann, and Schröder FLS11] (concurrent with our work), but it, like BGLS03, requires bilinear Diffie-Hellman.

Thus, the advantages of basing the schemes on trapdoor permutations (particularly a more standard security assumption and fast verification using lowexponent RSA) are offset by the disadvantage of requiring verification before signing. We argue below that this disadvantage is serious.

\subsection{The Need for Lazy Verification}

In applications with a large number of possible signers, the need to verify before signing can introduce a significant bottleneck, because each signer must retrieve the public keys of the previous signers before it can even begin to run its signing algorithm. Worse yet, signers need to keep their large caches of public keys secure and current: if a public key is revoked and a new one is issued, the signer must first obtain the new key and verify its certificate before adding its own signature to the aggregate.

A Key Application: BGPsec. Sequential aggregate signatures are particularly well-suited for the BGPsec Lep12 (formerly known as the Secure Border Gateway Protocol (S-BGP) [KLS00], a protocol being developed to improve the security of the global Internet routing system. (This application was mentioned in several works, including BGLS03LOS+06 Nev08, and explored further in [ZNN05.) In BGPsec, autonomous systems (ASes) digitally sign routing announcements listing the ASes on the path to a particular destination. An announcement for a path that is $n$ hops long will contain $n$ digital signatures, added in sequence by each AS on the path. (Notice that the length of the BGPsec message even without the signatures increases at every hop, as each AS adds its name to the path, as well as extra information to the material in the routing message like its "subject key identifier" - a cryptographic fingerprint that is used to lookup its public key in the PKI Lep12.) The BGPsec protocol is faced with two key performance challenges:

1. Obtaining public keys. BGPsec naturally requires routers to have access to a large number of public keys; indeed, a routing announcement can contain information from any of the 41,000 ASes in the Internet COZ08, (this number is according to the dataset retrieved in 2012). Certificates for public keys are regularly rolled over to maintain freshness, and must be retrieved from a distributed PKI infrastructure Hus12. Caching more than 41,000 public keys is expensive for a memory-constrained device like a router (which often does not have a hard drive or other secondary storage [KR06]). Furthermore, whenever a router sees a BGPsec message containing a key that is not in 
its cache, it incurs non-trivial delay on certificate retrieval (from a distant device that hosts the PKI) and verification.

2. Dealing with routing table "dumps". When a link from a router to its neighboring router fails, the router receives a dump of the full routing table, often containing more than 300,000 routes [CID, from it neighbors. Because routers are CPU- and memory-constrained devices, dealing with these huge routing table dumps incurs long delays (up to a few minutes, even with plain, insecure BGP [BHMT09]!). The delays are exacerbated if cryptographic signing and verifying is added to the process, and even more so when a router comes online for the first time (or after failure) and needs to also retrieve and authenticate public keys for all the ASes on the Internet.

To deal with these issues, the BGPsec protocol gives a router the option to perform lazy verification: that is, to immediately sign the routing announcement with its own public key, and to delay verification until a later time, e.g., when (a) it has time to retrieve the public keys of the other signers, or (b) when the router itself is less overloaded and can devote resources to verification [DHS]. It is important to note that lazy verification by one router need not hurt others: if a router has not verified a given announcement, routers further in the chain can verify it for themselves.

While there is legitimate concern that permitting lazy verification may cause routers to temporarily adopt unverified paths, the alternative may be worse: forbidding lazy verification can lead to problems with global protocol convergence (agreement on routes in the global Internet), because of routers that delay their announcements significantly until they can verify signatures (e.g., during routing table dumps, or while waiting to retrieve a missing certificate). Such delays create their own security issues, enabling easier denial of service attacks and traffic hijacking during the long latency window. Thus, even though BGPsec recommends that every router eventually verifies BGPsec messages, requiring that routers always verify before signing and re-announcing BGPsec messages is considered a nonstarter by the BGPsec working group [Sri12, Section 8.2.1]. Lazy verification is written into the BGPsec protocol specification as follows Lep12, Section 7]:

...it is important to note that when a BGPSEC speaker signs an outgoing update message, it is not attesting to a belief that all signatures prior to its are valid.

Requirement: No Public Keys in the Signing Algorithm! Note that the primary obstacle here is not only verification time (which can perhaps be improved through batching and, anyway, can be considerably faster than signing time when using low-exponent RSA), but also the need to obtain public keys. Thus, lazy verification also requires that prior signers' public keys are not used in the signing algorithm (e.g., hashed with the message as in [LMRS04 Nev08]). 


\section{Requirement: No Security Risk from Signing Unverified Aggregates!}

As we already mentioned, a signer who adds its own signature to an unverified aggregate in the schemes of [MRS04 and [Nev08 is exposed to a devastating attack. We already discussed how lazy verification may cause a signer to do so. Moreover, even without lazy verification, BGPsec may sometimes require a signer to add its own signature to an aggregate that is invalid. One such situation is when a router knowingly adopts a path that fails verification - for example, if it is the only path to a particular destination (the specification allows this Lep12. Section 5]). It will then add its own signature to the invalid one, because a "BGPSEC router should sign and forward a signed update to upstream peers if it selected the update as the best path, regardless of whether the update passed or failed validation (at this router)" Sri12, Section 8.2.1]. The need to sign a possibly invalid aggregate also arises in the case each message is signed by two different signature schemes (as will happen during transition times from one signature algorithm to another), and "one set of signatures verifies correctly and the other set of signatures fails to verify." In such a case the signer should still "add its signature to each of the [chains] using both the corresponding algorithm suite" [Lep12, Section 7]. Even if all BGPsec adopters avoid lazy verification and always verify before signing, these guidelines make it impossible to adopt an aggregate signature scheme that does not permit signing unverified aggregates, because of the possibility of attack. In other words, lazy verification is still needed for security even if no one uses it for efficiency!

Our Goal. We note that lazy verification is permitted by the trivial solution of concatenating individual ordinary signatures, by aggregate signature schemes defined in BGLS03, and by history-free aggregate signature schemes defined in [FLS11. All of the above schemes do not require the current signer to know anything about the previous signers: neither their public keys nor the messages they signed. 3 Our goal is to obtain the same advantages, while relying on a more basic security assumption than the bilinear Diffie-Hellman of [BGLS03|FLS11. and saving space as compared to the trivial solution.

${ }^{3}$ Identity-based aggregate signatures YCK04, XZF05, CLW05, CLGW06, Her06, GR06, BGOY07, HLY09, SVSR10, BJ10 also remove the need for obtaining public keys and have been proposed for use in BGPsec. However, agreeing on the secret-key-issuing authority for the global Internet seems politically infeasible. Moreover, on a technical level, the proposals either require interaction among signers or are based on bilinear pairings. Interactive signatures would significantly complicate the protocol. And if we are willing to rely on bilinear pairings, BGLS03, already gives us an excellent choice that allows for lazy verification.

Synchronized aggregate signatures (identity-based ones of [GR06 and regular ones of AGH10]) also allow for lazy verification, but require a common nonce for all signers that, if repeated, breaks the security of the scheme. Implementing such a nonce in BGPsec presents its own challenges, because each signer has to ensure it never reuses a nonce, or else its secret key is at risk. The schemes are also pairingbased. 


\subsection{Overview of Our Contributions}

We present a sequential aggregate signature scheme that is secure even with lazy verification, based on any trapdoor permutation (such as RSA). Moreover, as in the nonsequential scheme of BGLS03 and the history-free scheme of [FLS11, our signers do not need to know anything about each other - not even each other's public keys. To achieve this, we modify Neven's scheme Nev08 by randomizing the $H$-hash function with a fresh random string per signer, which becomes a part of the signature, similarly to Coron's PFDH Cor02 (Section 3). Our modification allows each signer to sign without verifying, and without even needing to know the public keys of all the signers that came before him, avoiding, in particular, the attack on LMRS04|Nev08.

Although the ultimate goal in aggregate signatures is to produce schemes whose signature length is independent of the number of signers, signatures in our scheme grow slightly with the number of signers. However (as also pointed out by [Nev08]), while a constant-length aggregate signature is a theoretically interesting goal, what usually matters in practice is the combined length of signatures and messages, because that's what verifiers receive: signatures rarely live on their own, separately from the messages they sign. And the combined length of messages, if they are distinct, grows linearly with the number of signers, so the total growth of the amount of information received by the verifier is anyway linear. What matters, then, is how fast this linear growth is; below we derive parameters that show it to be much smaller than when ordinary trapdoorpermutation-based signatures are used as in the trivial solution.

We make the following contributions:

Generic Randomized Scheme. We present the basic version of our scheme, which requires each signer to append a truly random string to the aggregate (Section 3). Our scheme is as efficient for signing and verifying (per signer) as ordinary trapdoor-permutation based signatures, like the Full-Domain-Hash (FDH, BR93, Section4]). We prove security (Section 4) in the random oracle model, based on the same assumption of trapdoor permutations (or claw-free permutations for a tighter security reduction) as in Nev08. Our security proof is more involved, because the reduction cannot know the public keys of other (adversarial) signers during the signature queries. We should note that our proof technique also shows that Neven's scheme need not hash other signer's public keys in the signing algorithm (however, Neven's scheme still fails under lazy verification).

Shortening the Randomness. We show that the per-signer random string can be shorter if it is made input-dependent (Section 5), ensuring that a given signer never produces two different signatures on the same input. The idea of inputdependent randomness has been used before in signature schemes (e.g., [KW03, Section 4]); however, our application requires a new combinatorial argument to show security.

Instantiating with RSA. In the full version of the paper BGR11b we show how to instantiate our schemes with practical trapdoor permutations like RSA, which have slightly different domains for different signers. 
Detailed Specification. We provide a full, parameterized step-by-step specification of the truly-random and input-dependent-random versions of our signature when instantiated with RSA (see the full version of the paper BGR11b, where we also provide guidelines on choosing parameters such as bit lengths).

Implementation, Benchmarking and Practical Considerations. We implement our specification as a module in OpenSSL (Section 6); the implementation is available from [BGR11a. We compare our implementation's performance to other potential solutions that allow for lazy verification; namely, BGLS03, and the "trivial" solution of using $n$ RSA or ECDSA signatures (the two algorithms currently proposed for use in implementations of BGPsec [DHS]). When evaluating signatures schemes for use with BGPsec, we consider compute time as well as signature length. Thus, we show that our signature is shorter than trivial RSA when there are $n>1$ signers and shorter than trivial ECDSA when there are $n>6$ signers. (While our signature is longer than the constant-length BGLS03 signature, it benefits from relying on the better-understood security assumption of RSA.) Moreover, our scheme enjoys the same extremely fast verify times as RSA.

\section{Preliminaries}

Sequential Aggregate Signature Security. The security definition for aggregate signatures (both original [BGLS03] and sequential [LMRS04]) is designed to capture the following intuition: each signer is individually secure against existential forgery following an adaptive chosen-message attack [GMR88] regardless of what all the other signers do. In fact, we will allow the adversary to give the attacked signer arbitrary - perhaps meaningless - aggregate-so-far signatures during the signature queries, thus making them adaptive "chosen-message-andaggregate" queries. We also allow the adversary, which we call "the forger," to choose the public keys of all the other signers and to place the single signer who is under attack anywhere in the signature chain in the attempted forgery. This single attacked signer does not know any public keys other than its own and does not verify any aggregate-so-far given by the attacker.

Our formal definition, presented in the full version [BGR11b], is almost verbatim from [LMRS04, with one important difference needed to enable lazy verification: the public keys and messages of previous signers are not input to the signing algorithm. Therefore, each signer, by signing a message, is attesting only to that message, not to the prior signers' messages and public keys. At a technical level, this change implies that in security game the forger, in its query to $i$ th signer, is required to supply only the aggregate-so-far signature allegedly produced by the first $i-1$ signers, but not the messages or public keys with respect to which this aggregate was allegedly produced. And, of course, to be considered successful, the forger must use a new message - in other words, it is not enough to change a public key or message of someone else in the chain before the attacked signer (because such public keys and messages may not even be well defined during the attack). This definition is exactly the one that is satisfied 
by the trivial solution of concatenating $n$ individual signatures (and therefore suffices, in particular, for BGPsec).

Fischlin, Lehmann, and Schröder [FLS11] propose a stronger security definition for their "history-free" signatures (building on history-free MACs of $\mathrm{EFG}^{+} 10$ ), which prevents certain reordering and recombining of signatures. Their definition thus has a security property that the trivial solution of concatenating $n$ individual signatures does not have. Although this security property is not needed in many applications (for example, in BGPsec reordering and recombining of signatures is prevented simply by the protocol message structure, where each message must, for the purposes of functionality, include all the signed information contained in previous messages), our signature scheme in fact also prevents reordering and recombining that are of concern to [FLS11]: see BGR11b.

Cryptographic Primitives. We will use pseudorandom functions GGM86; the definition is omitted here because it is standard, but is presented in [BGR11b] for the sake of completeness. We will denote by $\varepsilon_{\mathrm{PRF}}(q, t)$ the maximum insecurity of PRF against any distinguisher who asks at most $q$ queries and runs in time $t$.

We assume the reader is familiar with the trapdoor and claw-free permutations; we will denote by $\pi$ the easy direction of the trapdoor permutation, by $\pi^{-1}$ the hard direction, and by $\rho$ the function such that it is hard to find a "claw" $x, z$ with $\pi(x)=\rho(z)$.

\section{Our Basic Signature Scheme}

The intuition behind our construction is as follows. Like [Nev08, we use a random-oracle-based signature with message recovery, similar to PSS-R [BR96], as a basic building block. Signatures with message recovery embed a portion of the message into the signature, so it can be recovered on verification and does not need to be sent explicitly. In our case, the signature outputs two values: the output $x$ of a trapdoor permutation and an additional hash value $h$. The $i^{\text {th }}$ signer receives $\left(x_{i-1}, h_{i-1}\right)$ from the previous signer and wants to sign a message $m_{i}$. To enable aggregation, we view $\left(x_{i-1}, m_{i}\right)$ together as a "message" to be signed with message recovery: we apply the signature with message recovery to this pair, so that $x_{i-1}$ is embedded into the signature and does not have to be sent explicitly. The $h$ portions of the signatures are exclusive-ored together for aggregation.

So far, what we described is a slightly simplified version of the scheme from Nev08. Note that verifying before signing is necessary in this scheme, because the transformation from $\left(x_{i-1}, h_{i-1}\right)$ to $\left(x_{i}, h_{i}\right)$ is deterministic, invertible, and can be performed by the adversary, except for the inversion of the trapdoor permutation performed at the last step. As we show in [BGR11b, no scheme constructed in this manner can permit lazy verification while protecting against a chosen message attack. Thus, to enable lazy verification, we require each signer to add a random string to the message, and concatenate and append these strings to the signature. Because the adversary lacks a priori knowledge about these 
random strings, the chosen message attack becomes useless and we can prove that this is sufficient to enable lazy verification.

Notation. We now describe the scheme precisely, using the following notation:

- Let $m_{i}$ be the message signed by signer $i$.

- Let trapdoor permutation $\pi_{i}$ be the public key of signer $i$ and $\pi_{i}^{-1}$ be the corresponding secret key. We assume all permutations operate on bit strings of length $\ell_{\pi}$, i.e., have domain and range $\{0,1\}^{\ell_{\pi}}$. (In the full version BGR11b] we remove the assumption that all permutations operate on the same domain. Section 6 uses this to instantiate $\pi$ from the RSA assumption, where $\pi_{i}$ is the easy direction, and $\pi_{i}^{-1}$ is the hard direction of the RSA permutation.)

- Let $H($ resp. $G)$ be a cryptographic hash function (modeled as a random oracle) that outputs $\ell_{H}$-bit $\left(\right.$ resp.$\ell_{\pi}$-bit) strings.

- Let $\ell_{r}$ be a parameter denoting the length of the randomness appended by each signer.

- Let the notation $\boldsymbol{a}_{\boldsymbol{i}}$ denote a vector of values $\left(a_{1}, a_{2}, \ldots, a_{i}\right)$.

- Let $\oplus$ to denote bitwise exclusive-or. Exclusive-or is not the only operation that can be used; any efficiently computable group operation with efficient inverse can be used here.

$-\epsilon$ is a special character denoting the empty string; we assume $\epsilon \oplus x=x$ for any $x$.

Sign: The $i^{\text {th }}$ Signer's algorithm

\section{Require:}

$$
\begin{aligned}
& \pi_{i}, \pi_{i}^{-1}, m_{i}, x_{i-1}, h_{i-1} \\
& \left(\text { where } x_{i-1}, h_{i-1}=\epsilon, \epsilon\right. \text { if } \\
& i=1 \text { ). }
\end{aligned}
$$

1: Draw $r_{i} \stackrel{R}{\leftarrow}\{0,1\}^{\ell_{r}}$

2: $\eta_{i} \leftarrow H\left(\pi_{i}, m_{i}, r_{i}, x_{i-1}\right)$

3: $h_{i} \leftarrow h_{i-1} \oplus \eta_{i}$

4: $g_{i} \leftarrow G\left(h_{i}\right)$

5: $y_{i}=g_{i} \oplus x_{i-1}$

6: $x_{i} \leftarrow \pi_{i}^{-1}\left(y_{i}\right)$

7: return $r_{i}, x_{i}, h_{i}$ \{Note that $x_{i}$ and $h_{i}$ go to the next signer; all the $r_{i}$ values go to the verifier, but only the last signer's $x_{i}$ and $h_{i}$ do. $\}$
$\operatorname{Ver}^{H, G}$ : The Verification Algorithm

Require: $\boldsymbol{\pi}_{\boldsymbol{n}}, \boldsymbol{m}_{\boldsymbol{n}}, \boldsymbol{r}_{\boldsymbol{n}}, x_{n}, h_{n}$

1: for $i=n, n-1, \ldots, 2$ do

2: $\quad y_{i} \leftarrow \pi_{i}\left(x_{i}\right)$

3: $\quad g_{i} \leftarrow G\left(h_{i}\right)$

4: $\quad x_{i-1} \leftarrow g_{i} \oplus y_{i}$

5: $\quad \eta_{i} \leftarrow H\left(\pi_{i}, m_{i}, r_{i}, x_{i-1}\right)$

6: $\quad h_{i-1} \leftarrow h_{i} \oplus \eta_{i}$

7: if $h_{1}=H\left(\pi_{1}, r_{1}, m_{1}, \epsilon\right)$ and $\pi_{1}\left(x_{1}\right)=G\left(h_{1}\right)$ then

8: return 1

9: else

10: return 0

The $i^{\text {th }}$ signer's signing algorithm has no dependency on the number of signers; it takes in only the $i^{\text {th }}$ signers' own public key and message and the aggregated portion of the signature $x_{i-1}, h_{i-1}$. Moreover, the aggregated signature need not be verified before it is signed. For verification, only a single $x_{i}$ and $h_{i}$-namely, the one from the last signer - is needed. However, every $r_{i}$, from the first signer to the last, is needed. 


\section{Security Proof}

We prove our scheme secure if $G$ and $H$ are modeled as random oracles and $\pi$ is a trapdoor permutation. The proof is easier to understand if $\pi$ is additionally claw-free (in particular, any homorphic permutation, such as RSA, is claw-free if it is trapdoor). We therefore present the proof for the claw-free case. The more general case is addressed in the full version BGR11b. Our proof shows how a forger $F$ on the aggregate signature scheme can be used to construct a reduction $R$ that finds a claw in claw-free pair $\left(\pi_{*}, \rho_{*}\right) . R$ has $F$ forge a signature for victim signer that uses permutation $\pi_{*}$, and then uses the resulting forgery to find the claw in the claw-free pair. The structure of our reduction is similar to Nev08; however, while [Nev08 constructs a "sequential forger" from forger $F$ and then constructs reduction $R$ from the sequential forger, our reduction must proceed in one step (since the notion of a sequential forger is undefined if hash queries do not include previous signers public keys).

$F$ 's Queries. We review what forger $F$ expects to see on each one of its queries:

- H-Query. $F$ asks query $Q=(\pi, m, r, x)$ (where $x$ may be $\epsilon$ ) and expects to see $H(Q)=\eta$.

- G-query. $F$ asks query $h$, and expects to see $g=G(h)$.

- Sign Query. $F$ asks query $(m, h, x)$ to be signed by $\pi_{*}$, and expects to see $r, h^{\prime}, x^{\prime}$ back, where $r$ looks uniform, $h^{\prime}=h \oplus H\left(\pi_{*}, m, r, x\right)$, and $\pi_{*}\left(x^{\prime}\right)=$ $G\left(h^{\prime}\right) \oplus x$.

- Forgery. Finally, $F$ outputs a forgery, $\sigma=\boldsymbol{\pi}_{\boldsymbol{n}}, \boldsymbol{m}_{\boldsymbol{n}}, \boldsymbol{r}_{\boldsymbol{n}}, x_{n}, h_{n}$ where $\pi_{n}=$ $\pi_{*}$. (Value $n$ is chosen by $F$ ).

Simplifying Assumptions about the Forger $F$. The following simplifies our proof:

- We assume that the forger $F$ forges the last signature in the signature chain; in other words, $\pi_{n}=\pi_{*}$ and $m_{n}$ is a new message never queried by $F$ to the signing oracle (whose public key is $\pi_{*}$ ). Indeed, any $F$ can be easily modified to do so: if $\pi_{*}$ and a new message $m_{n^{\prime}}$ are present in $\pi_{n}$ but at location $n^{\prime}<n$, then we can run the verification algorithm loop for $n-n^{\prime}$ iterations to obtain $x_{n^{\prime}}, h_{n^{\prime}}$ and output $\sigma^{\prime}=\boldsymbol{\pi}_{\boldsymbol{n}^{\prime}}, \boldsymbol{m}_{\boldsymbol{n}^{\prime}}, \boldsymbol{r}_{\boldsymbol{n}^{\prime}}, x_{n^{\prime}}, h_{n^{\prime}}$ as the new forgery, which will be valid if an only if $\sigma$ was valid. Note that we do not assume that $\pi_{*}$ (or any other public key) is present in the signature chain only once.

- We assume that before forger $F$ outputs its forgery and halts, it makes hash queries on all the hashes that will be computed during the verification of its forgery. Moreover, we assume that the forger does not output an invalid forgery; instead, it halts and outputs $\perp$. Indeed, any $F$ can be modified to do so; simply run the verification algorithm upon producing the forgery, and check that $m_{n}$ is different from every message asked in a sign query.

\subsection{Description of the Reduction $R$}

Data Structures Used by $\boldsymbol{R}$. HT and GT Tables. The reduction $R$ uses 'programmable random oracles', i.e., it chooses answers for random oracle 
queries. $R$ keeps track of queries whose answers have already been decided in two tables: HT for $H$ and GT for $G$. We say $\mathrm{HT}(Q)=\eta$ if $\mathrm{HT}$ stores $\eta$ as the answer to a query $Q$, and $\mathrm{HT}(Q)=\perp$ if $\mathrm{HT}$ has no answer for $Q$ (similar for GT).

The HTree. The key challenge for the reduction is programming $G$, since $G$ queries are made on sums of $H$-query answers, rather than on individual $H$-query answers. Thus the reduction keeps an additional data structure, the HTree, that records responses to $H$-queries that may eventually be used as part of forger $F$ 's forgery. (HTree is inspired by the graph $\mathcal{G}$ in Nev08, Lemma 5.3].)

The HTree is a tree of labeled nodes that stores a subset of the queries in HT. Each node in HTree (except the root) corresponds to an $H$-query that could potentially appear in the forger $F$ 's final forgery $\sigma$; the queries asked during verification of $\sigma$ will appear on a path from one of the leaf nodes to the root (unless a very unlikely event occurs). The HTree has a designated root node that stores the value $h_{0}=0$. We consider the root to be at depth 0 . A node $N_{i}$ at depth $i>0$ stores:

- a pointer to its parent node

- a query $Q_{i}=\left(\pi_{i}, m_{i}, r_{i}, x_{i-1}\right)$ (where $x_{i-1}=\epsilon$ if and only if $i=1$ ),

- the 'hash-response' values $\eta_{i}$ and $h_{i}$ ( $h_{i}$ is the XOR of the values $\eta_{1}, \ldots, \eta_{i}$ on the path from the root to the node $N_{i}$; equivalently, $h_{i-1} \oplus \eta_{i}$, where $h_{i-1}$ is stored in the parent node),

- an auxiliary value $y_{i}$ that is used to determine how future queries are added to the HTree, computed as $G\left(h_{i}\right) \oplus x_{i-1}$ (note that $y_{i}$ is the value to which the signer would apply $\left.\pi_{i}^{-1}\right)$,

- if $\pi_{i}=\pi_{*}$, an auxiliary value $z$ that may be used to find a claw in $\left(\pi_{*}, \rho_{*}\right)$.

Every node at depth $i=2$ or deeper satisfies the relation $\pi_{i-1}\left(x_{i-1}\right)=y_{i-1}$, where $\pi_{i-1}$ and $y_{i-1}$ are stored at the node's parent. New $H$-queries $Q$ are added as nodes to the HTree if they can satisfy this relation; we say that such a query can be tethered to an existing node in the HTree. Intuitively, a query tethered to $N_{i}$ becomes a child of $N_{i}$ in the HTree:

Definition 1 (Tethered queries). An $H$-query $Q$ containing $x \neq \epsilon$ is tethered to node $N_{i}$ in the HTree if $N_{i}$ stores $\pi_{i}, y_{i}$ such that $\pi_{i}(x)=y_{i}$. If $x=\epsilon$, then $Q$ is tethered to the root of the HTree.

The HTree's Lookup function determines the HTree node to which query $Q$ can be tethered. We can argue that Lookup finds at most one node with high probability.) The HTree is populated via the Sim-H algorithm. The reduction $R$ adds an $H$-query $Q$ to the HTree if and only if it is tethered to some node in the HTree at the time that forger $F$ makes the $H$-query. It is possible that some query $Q$ is not tethered at the time it is made, but becomes tethered at at later time (after some new nodes are added to the HTree). However, we show that this is highly unlikely. 
Algorithms Used Used by $\boldsymbol{R}$. The reduction $R$ uses the following algorithms, which are formally specified in the full version BGR11b.

$G$-Queries. $R$ answers these queries using a simple algorithm Sim-G. Sim-G returns $\mathbf{G T}(h)$ if it is already defined, or, if not, returns a fresh random value and records it in the GT.

Sign-Queries. The reduction $R$ answers queries $(m, h, x)$ to be signed by $\pi_{*}$ using Sim-S. Since the reduction does not know the inverse of the challenge permutation $\pi_{*}^{-1}$, it 'fakes' a valid signature by carefully assigning certain entries in random oracle tables $\mathrm{HT}, \mathrm{GT}$, and ABORTS if these entries in HT, GT have been previously assigned. We are able to argue that Sim-S is unlikely to abort, since the entries added to HT, GT by Sim-S depend on a fresh random value $r$ chosen as part of each signature query.

$H$-Queries. The reduction $R$ answers these queries $Q=(\pi, m, r, x)$ using $\mathrm{Sim}-\mathrm{H}$. If there is an entry for $Q$ in the $\mathrm{HT}$, then Sim- $\mathrm{H}$ returns it. Otherwise, it assigns a fresh random value $\eta$ as $\mathrm{HT}(Q)$. Next, Sim- $\mathrm{H}$ needs to prepare for the event that $Q$ could lead to a forgery by the forger $F$, and thus needs to be stored in the HTree. To do this, Sim-H uses the Lookup function to check if $Q$ can be tethered and thus should be added to the HTree. If $Q$ can be tethered, Sim-H adds a new node to the $\mathrm{HTree}$ containing $Q$, its hash response $\eta$, and an auxiliary value $y$ that is used by the Lookup function to tether future $H$-queries. In order to ensure that HTree is a tree, it is important to ensure that $y$ is a fresh random value; Sim- $\mathrm{H}$ aborts if that's not the case. Finally, if $Q$ contains the challenge permutation $\pi_{*}$, Sim- $\mathrm{H}$ adds a value $z$ to the $\mathrm{HTree}$ node that FindClaw will use to derive a claw from a valid forgery output by the forger $F$. To prepare these values, Sim- $\mathrm{H}$ behaves almost as if it is 'faking' the answer to a sign-query, except that instead of using the usual challenge permutation $\pi_{*}$ (as in Sim-S), it uses the challenge permutation $\rho_{*}$ applied to $z$ (so as to benefit from forger $F$ 's forgery, which would invert $\pi_{*}$ on the output of $\rho_{*}(z)$, thus producing a claw). As in Sim-S, this involves carefully assigning certain entries in GT, and aborting if these entries are already assigned. We are able to show that Sim-H is unlikely to abort.

Finding a Claw. Finally, forger $F$ outputs a forgery $\boldsymbol{\pi}_{\boldsymbol{n}}, \boldsymbol{m}_{\boldsymbol{n}}, \boldsymbol{r}_{\boldsymbol{n}}, x_{n}, h_{n}$, where $\pi_{n}=\pi_{*}$. Recall that our simplifying assumptions mean that the forgery is valid. The reduction $R$ uses FindClaw to find a claw from the forgery. Because we assumed all the queries for verifying $\sigma$ have already been asked, the query $\left(\pi_{*}, m_{n}, r_{n}, x_{n-1}\right)$ is in HT. Moreover, if the forgery is valid, then with high probability it is in the HTree as a child of the node storing $\left(\pi_{n-1}, m_{n-1}, r_{n-1}, x_{n-2}\right)$, which is in turn a child of the node storing $\left(\pi_{n-2}, m_{n-2}, r_{n-2}, x_{n-3}\right)$, etc. This holds because in a valid forgery, each $H$-query made during verification is tethered to the next one, and all tethered queries are in the HTree with high probability. The value $x_{n}$ (from the forgery $\sigma$ ) and value $z_{n}$ (from $\mathrm{HTree}$ node of the query $\left.Q=\left(\pi_{*}, m_{n}, r_{n}, x_{n-1}\right)\right)$ constitute a claw. 


\subsection{Analysis of the Reduction}

Theorem 1. If a forger $F$ succeeds with probability $\varepsilon$, then the reduction $R$ finds a claw for $\left(\pi_{*}, \rho_{*}\right)$ in about the same running time as $F$ with probability

$$
\varepsilon-\left(q_{S}+q_{H}\right)\left(q_{S}+q_{G}+q_{H}\right) 2^{-\ell_{H}}-q_{S}\left(q_{S}+q_{H}\right) 2^{-\ell_{r}}-q_{H}^{2} 2^{-\ell_{\pi}}
$$

where $q_{H}$ is the number of $H$-hash queries, $q_{G}$ is the number of $G$-hash queries, and $q_{S}$ is the number of sign queries made by the forger $F$.

We prove this theorem in full version of the paper BGR11b. The proof hinges on two key statements about the HTree. First, the probability that Lookup $(x)$ finds more than one HTree node is low (even though Lookup uses the functions $\pi$ stored in the nodes of the HTree, which do not have to be permutations, because they are adversarially supplied and not certified like in [LMRS04]). Second, an $H$-query that was not added to HTree is unlikely to become tethered at some later time. Both statements rely on the fact that each time a query is placed on the HTree, its $y$ value is random and independent of every other $y$ value.

\section{Shorter Signatures via Input-Dependent Randomness}

To shorten our signature, we now show how to reduce $\ell_{r}$ (the length of the randomness appended by each signer). To do this, we replace the truly random $r$ from our basic scheme with an $r$ that is computed as a function of the inputs to the signer, and argue that it can be made shorter than the random $r$. Intuitively, we are able to maintain security with a shorter $r$ because a given signer never produces two different signatures on the same input, thus limiting the information that an adversary can see and exploit. Of course, this input-dependent $r$ need not be truly random; it suffices for a $r$ to be a pseudorandom function of the input.

\subsection{Modifying the Scheme}

We now compute $r$ as a pseudorandom function (PRF) over the input $\left(m_{i}\right.$, $\left.h_{i-1}, x_{i-1}\right)$ received by that signer $i$. Let $\mathrm{PRF}_{\text {seed }}:\{0,1\}^{*} \rightarrow\{0,1\}^{\ell_{r}}$ be a PRF with seed seed and insecurity $\varepsilon_{\mathrm{PRF}}(q, t)$ against adversaries asking $q$ queries and running in time $t$. Add a uniformly chosen seed to the secret key of the signer and replace line 1 of the signing algorithm with $r \leftarrow \mathrm{PRF}_{\text {seed }}(m, h, x)$.

In the previous section, we found that $\ell_{r}$ must be long enough to tolerate a security loss of $q_{S}\left(q_{H}+q_{S}\right) 2^{-\ell_{r}}$ (Theorem 11). As we show below, $\ell_{r}$ in the modified scheme can be shorter, since it needs only to allow for a security loss of approximately $\left(q_{G}+q_{H}+q_{S}+\ell_{H} q_{S}^{2}\right) 2^{-\ell_{r}}$. This is an improvement if we assume that $q_{H} \approx q_{G}$ (since both $H$ and $G$ are hash functions) and $q_{S} \ll q_{H}$ (since in practice hash queries can be made offline, while signing queries need access to an actual signer). 


\subsection{Key Insight for the Security Proof}

Using the reduction of Section 4, we had to choose $r$ long enough to make it unlikely that when a forger makes a sign query on $\left(\pi_{*}, m_{i}, x_{i-1}, h_{i-1}\right)$, the algorithm Sim-S draws a random $r_{i}$ that collides with a previously made $\mathrm{H}$ query $Q_{i}=\left(\pi_{*}, m_{i}, r_{i}, x_{i-1}\right)$. Indeed, if $Q_{i}$ was answered by $\eta_{i}$ and the forger chooses $h_{i-1}$ so that $h_{i}$ (which is computes as $h_{i-1} \oplus \eta_{i}$ ) has already been queried to $G$, then when $r$ collides, the reduction would be prevented from programming the random oracle $G\left(h_{i}\right)$. Making $r$ depend on the forger's input to the signer means that the forger gets only one chance (rather than $q_{S}$ chances) to make this happen for a given $Q_{i}, h_{i-1}$, and $h_{i}$, because subsequent attemps by the forger will use the same $r$.

We show in the full version BGR11b that the problem of proving this modified scheme secure hinges on the following combinatorial problem.

Combinatorial Problem. Suppose $\beta$ values $\eta_{1}, \ldots, \eta_{\beta}$ are chosen uniformly at random as $\ell_{H^{-}}$bit strings and given to an adversary, who then chooses $\alpha$ distinct values $h_{1}^{\prime}, \ldots, h_{\alpha}^{\prime}$. The $\alpha \times \beta$-matrix $\zeta$ is constructed by XORing the $\eta$ and the $h^{\prime}$ values. A collision in $\zeta$ is a set of entries that are all equal. What is the total number of entries in the $\gamma$ biggest collisions?

Theorem 2. With probability at least $1-\beta^{2} 2^{\ell_{H}}$, the total size of the $\gamma$ biggest collisions in $\zeta$ is at most $\alpha+\left(\ell_{h}+2\right) \gamma^{2}$.

The proof of this theorem, as well as the entire security analysis of the modified scheme, are found in BGR11b.

\section{Implementation and Evaluation}

In the full version of the paper BGR11b we present details of instantiating our scheme with RSA (these include, in particular, dealing with the problem of slightly different domains for each signer's permutation). We implemented the input-dependent- $r$ version as a module in OpenSSL [ope]. The code is available from BGR11a.

Overview of Our Implementation. We instantiate the permutation $\pi$ with 2048-bit RSA with public exponent 65537, hash $H$ with SHA-256, full-domain hash $G$ with the industry-standard Mask Generating Function (MGF) using SHA-256 [RSA02, and the pseudorandom function PRF with HMAC-SHA-256 BCK96. Instead of hashing the permutation $\pi$ as-is inside the hash function $H$, we replace it with a short fingerprint of the RSA public key computed using SHA-256. Thus, we have parameters $\ell_{\pi}=2048, \ell_{h}=256$, and $\ell_{r}=128$; the $\ell_{r}$ value is per signer, and each signer also adds one bit of information to deal with the problem that RSA gives each signer a slightly different domain. Therefore, the length of the aggregate signature for $n$ signers is $2048+256+129 n$ bits long (see Table 11). We justify this choice of parameters in [BGR11b]. 
Table 1. Benchmark results for $n$ signers. Computed on a laptop with a Core i3 processor at $2.4 \mathrm{GHz}$ and $2 \mathrm{~GB}$ RAM, running Ubuntu. The first three schemes were implemented using OpenSSL ope] (with SHA-256 hashing and RSA public exponent of 65537); the BGLS scheme was implemented using MIRACL [Sco11] (with the curve BN128 [BN05] and with precomputation on the curve generator but not on the public keys; further precomputation on the public keys seems to improve verification performance by up to $20 \%$ at the cost of additional storage). Results for specific values of $n$ are not exactly in proportion due to rounding.

\begin{tabular}{|c|c|c|c|c|}
\hline & 2048-bit RSA & Our scheme & 256-bit ECDSA & 256-bit BGLS \\
\hline \hline Signature length (bits) & $2048 n$ & $2304+129 n$ & $512 n$ & 257 \\
\hline Length for $n=4.5$ & 9216 & 2885 & 2304 & 257 \\
\hline Length for $n=7$ & 14336 & 3207 & 3584 & 257 \\
\hline Sign time $(\mathrm{ms})$ & 11.8 & 11.9 & 2.3 & 1.9 \\
\hline Verify time $(\mathrm{ms})$ & $0.3 n$ & $0.3 n$ & $2.8 n$ & $\approx 18.9+6.6 n$ \\
\hline Verify time for $n=4.5$ & 1.3 & 1.3 & 12.5 & 47.6 \\
\hline Verify time for $n=7$ & 2.1 & 2.1 & 19.4 & 64.8 \\
\hline
\end{tabular}

Evaluation. We compare the implementation described in the previous paragraph to other signature schemes that allow for lazy verification. Table 1 contains data on our scheme as well as the "trivial" solution of using $n$ RSA signatures, the solution of similarly using $n$ ECDSA Van92 IEE02 signatures (which are current contenders for adoption in BGPsec [Sri12, Section 4.1]), and the aggregate scheme of BGLS03] (we do not compare against FLS11, because it is a more complicated version of [BGLS03, so [BGLS03] performs better than [FLS11], anyway). In addition to providing formulas in terms of the number $n$ of signers, we show results for specific values of $n=4.5$ and $n=7$. The value of 4.5 was chosen because it is roughly the average length of an AS path for a well-connected router on the Internet today (average length fluctuates with time and vantage point - see, e.g., here [Smi12]). We should note, however, that performance for higher than average values of $n$ is particularly important: transition to BGPsec is expected to be particularly problematic for weaker routers, which are more likely to be located to in the less well-connected portions of the Internet, and that experience longer than average paths. We therefore also show results for $n=7$.

The table shows that the BGLS03 scheme is a clear winner in terms of signature length and signing time, but has considerably slower verification 4 . It should be noted, however, that it is not being considered for the BGPsec standard at this stage [Sri12, Section 4.1]: schemes relying on bilinear DiffieHellman are not considered ready for worldwide deployment on the internet backbone by the BGPsec working group, because a consensus has not emerged on which curves provide the right tradeoff between security and efficiency (for example, there is not a NIST-approved set of curves such as the one contained in [NIS09, Appendix D] for non-pairing-based elliptic-curve cryptography). It

\footnotetext{
${ }^{4}$ A more efficient pairing-based scheme of WM08 with a constant total number of pairings was shown insecure by $\mathrm{SVS}^{+} 09$.
} 
is also important to note that the time required to compute group operations and bilinear pairings depends very heavily on the curve used; improvements for various curves are produced frequently, and there is no generally accepted set of curves or algorithms at this point. We believe that, assuming continued progress to speed-up pairings on specific curves and sufficient confidence in the security of bilinear Diffie-Hellman on these curves, the scheme of [BGLS03] (as improved by BNN07) should be considered for real applications.

As far as the remaining three schemes are concerned, we observe that ECDSA provides the shortest signatures when $n<6$, while our scheme dominates the three for $n>6$ (as we already mentioned, performance for higher than average $n$ is particularly important.) We also observe that our scheme has computation time almost identical to simple RSA while having much shorter signatures (RSA signature length is listed as a particular concern in [Sri12, Section 4.1.2]). While ECSDA has the fastest signing time, the verification times for RSA and our scheme are an order of magnitude faster than those of ECDSA. Note that, for a router, the time required to sign does not depend on $n$, but the time required to verify grows linearly with $n$, so verification times are also of particular importance to weaker routers at the edge of the network.

Thus, if one is interested in a scheme based on the standard assumption of trapdoor permutations (albeit in the random oracle model), then our proposal fits the bill. Moreover, even if one is willing to accept security of ECDSA (which is not known to follow from any standard assumptions), our scheme may be preferable based on fast verify times and comparable-length signatures. Our scheme also has much faster verifying that pairing-based BGLS.

Acknowledgements. We thank Anna Lysyanskaya for help with the early stages of this work, Hovav Shacham and Craig Costello for helpful pointers and explanations, and the DHS S\&T CSD Secure Routing project for many useful discussions that informed the design of our schemes. We thank anonymous referees for helping us improve our presentation and for pointing out related work. This work was supported by NSF Grants 1017907, 0546614, 0831281, 1012910, 1012798, and a gift from Cisco.

\section{References}

AGH10. Ahn, J.H., Green, M., Hohenberger, S.: Synchronized aggregate signatures: new definitions, constructions and applications. In: ACM Conference on Computer and Communications Security (2010)

BCK96. Bellare, M., Canetti, R., Krawczyk, H.: Keying Hash Functions for Message Authentication. In: Koblitz, N. (ed.) CRYPTO 1996. LNCS, vol. 1109, pp. 1-15. Springer, Heidelberg (1996)

BGLS03. Boneh, D., Gentry, C., Lynn, B., Shacham, H.: Aggregate and Verifiably Encrypted Signatures from Bilinear Maps. In: Biham, E. (ed.) EUROCRYPT 2003. LNCS, vol. 2656, pp. 416-432. Springer, Heidelberg (2003) 
BGOY07. Boldyreva, A., Gentry, C., O'Neill, A., Yum, D.H.: Ordered multisignatures and identity-based sequential aggregate signatures, with applications to secure routing. In: ACM Conference on Computer and Communications Security, pp. 276-285. ACM (2007)

BGR11a. Brogle, K., Goldberg, S., Reyzin, L.: Implementation of sequential aggregate signatures with lazy verification (2011),

http://www.cs.bu.edu/fac/goldbe/papers/bgpsec-sigs.html

BGR11b. Brogle, K., Goldberg, S., Reyzin, L.: Sequential aggregate signatures with lazy verification, Full version and implementation code (2011), http://www.cs.bu.edu/fac/goldbe/papers/bgpsec-sigs.html

BHMT09. Houidi, Z.B., Meulle, M., Teixeira, R.: Understanding slow bgp routing table transfers. In: Proc. ACM SIGCOMM Internet Measurement Conference, pp. 350-355. ACM, New York (2009)

BJ10. Bagherzandi, A., Jarecki, S.: Identity-Based Aggregate and MultiSignature Schemes Based on RSA. In: Nguyen, P.Q., Pointcheval, D. (eds.) PKC 2010. LNCS, vol. 6056, pp. 480-498. Springer, Heidelberg (2010)

BN05. Barreto, P.S.L.M., Naehrig, M.: Pairing-Friendly Elliptic Curves of Prime Order. In: Preneel, B., Tavares, S. (eds.) SAC 2005. LNCS, vol. 3897, pp. 319-331. Springer, Heidelberg (2006)

BNN07. Bellare, M., Namprempre, C., Neven, G.: Unrestricted Aggregate Signatures. In: Arge, L., Cachin, C., Jurdziński, T., Tarlecki, A. (eds.) ICALP 2007. LNCS, vol. 4596, pp. 411-422. Springer, Heidelberg (2007)

BR93. Bellare, M., Rogaway, P.: Random oracles are practical: A paradigm for designing efficient protocols. In: ACM Conference on Computer and Communications Security, pp. 62-73 (1993)

BR96. Bellare, M., Rogaway, P.: The Exact Security of Digital Signatures - How to Sign with RSA and Rabin. In: Maurer, U.M. (ed.) EUROCRYPT 1996. LNCS, vol. 1070, pp. 399-416. Springer, Heidelberg (1996)

CHKM10. Chatterjee, S., Hankerson, D., Knapp, E., Menezes, A.: Comparing two pairing-based aggregate signature schemes. Des. Codes Cryptography 55(2-3), 141-167 (2010)

CID. The CIDR report, http://www.cidr-report.org

CLGW06. Cheng, X., Liu, J., Guo, L., Wang, X.: Identity-based multisignature and aggregate signature schemes from $m$-torsion groups. Journal of Electronics (China) 23(4) (July 2006)

CLW05. Cheng, X., Liu, J., Wang, X.: Identity-Based Aggregate and Verifiably Encrypted Signatures from Bilinear Pairing. In: Gervasi, O., Gavrilova, M.L., Kumar, V., Laganá, A., Lee, H.P., Mun, Y., Taniar, D., Tan, C.J.K. (eds.) ICCSA 2005. LNCS, vol. 3483, pp. 1046-1054. Springer, Heidelberg (2005)

Cor02. Coron, J.-S.: Optimal Security Proofs for PSS and Other Signature Schemes. In: Knudsen, L.R. (ed.) EUROCRYPT 2002. LNCS, vol. 2332, pp. 272-287. Springer, Heidelberg (2002)

COZ08. Chi, Y.-J., Oliveira, R., Zhang, L.: Cyclops: The Internet AS-level observatory. In: ACM SIGCOMM CCR (2008)

CSC09. Chakrabarti, S., Chandrasekhar, S., Singhal, M., Calvert, K.L.: An efficient and scalable quasi-aggregate signature scheme based on lfsr sequences. IEEE Trans. Parallel Distrib. Syst. 20(7), 1059-1072 (2009)

DHS. Department of Homeland Security, Science and Technology Directorate, Cyber Security Division, Secure Protocols for Routing Infrastructure project. Personal Communication 
EFG $^{+}$10. $\quad$ Eikemeier, O., Fischlin, M., Götzmann, J.-F., Lehmann, A., Schröder, D., Schröder, P., Wagner, D.: History-Free Aggregate Message Authentication Codes. In: Garay, J.A., De Prisco, R. (eds.) SCN 2010. LNCS, vol. 6280, pp. 309-328. Springer, Heidelberg (2010)

FLS11. Fischlin, M., Lehmann, A., Schröder, D.: History-free sequential aggregate signatures. Technical Report 2011/231, Cryptology ePrint archive (2011), http://eprint.iacr.org

GGM86. Goldreich, O., Goldwasser, S., Micali, S.: How to construct random functions. J. ACM 33(4), 792-807 (1986)

GMR88. Goldwasser, S., Micali, S., Rivest, R.L.: A digital signature scheme secure against adaptive chosen-message attacks. SIAM J. Comput. 17(2), 281308 (1988)

GR06. Gentry, C., Ramzan, Z.: Identity-Based Aggregate Signatures. In: Yung, M., Dodis, Y., Kiayias, A., Malkin, T. (eds.) PKC 2006. LNCS, vol. 3958, pp. 257-273. Springer, Heidelberg (2006)

Her06. Herranz, J.: Deterministic identity-based signatures for partial aggregation. Comput. J. 49(3), 322-330 (2006)

HLY09. Hwang, J.Y., Lee, D.H., Yung, M.: Universal forgery of the identity-based sequential aggregate signature scheme. In: Li, W., Susilo, W., Tupakula, U.K., Safavi-Naini, R., Varadharajan, V. (eds.) ASIACCS, pp. 157-160. ACM (2009)

Hus12. Huston, G. (ed.): The Profile for Algorithms and Key Sizes for Use in the Resource Public Key Infrastructure (RPKI). IETF RFC 6485 (February 2012), http://tools.ietf .org/html/rfc6485

IEE02. IEEE Std 1363-2000. IEEE standard specifications for public-key cryptography (2002)

KLS00. Kent, S., Lynn, C., Seo, K.: Secure border gateway protocol (S-BGP). J. Selected Areas in Communications 18(4), 582-592 (2000)

KR06. Karpilovsky, E., Rexford, J.: Using forgetful routing to control bgp table size. In: Proceedings of the 2006 ACM CoNEXT Conference, CoNEXT 2006, pp. 2:1-2:12. ACM, New York (2006)

KW03. Katz, J., Wang, N.: Efficiency improvements for signature schemes with tight security reductions. In: Jajodia, S., Atluri, V., Jaeger, T. (eds.) ACM Conference on Computer and Communications Security, pp. 155164. ACM (2003)

Lep12. Lepinski, M. (ed.): BGPSEC Protocol Specification. IETF Network Working Group, Internet-Draft (July 2012),

http://tools.ietf .org/html/draft-ietf-sidr-bgpsec-protocol-04

LMRS04. Lysyanskaya, A., Micali, S., Reyzin, L., Shacham, H.: Sequential Aggregate Signatures from Trapdoor Permutations. In: Cachin, C., Camenisch, J. (eds.) EUROCRYPT 2004. LNCS, vol. 3027, pp. 74-90. Springer, Heidelberg (2004)

LOS+06. Lu, S., Ostrovsky, R., Sahai, A., Shacham, H., Waters, B.: Sequential Aggregate Signatures and Multisignatures Without Random Oracles. In: Vaudenay, S. (ed.) EUROCRYPT 2006. LNCS, vol. 4004, pp. 465-485. Springer, Heidelberg (2006)

Nev08. Neven, G.: Efficient Sequential Aggregate Signed Data. In: Smart, N.P. (ed.) EUROCRYPT 2008. LNCS, vol. 4965, pp. 52-69. Springer, Heidelberg (2008)

NIS09. FIPS publication 186-3: Digital signature standard (DSS) (June 2009), http://csrc.nist.gov/publications/PubsFIPS.html 
ope. OpenSSL toolkit, http://openssl.org/

RS09. Rückert, M., Schröder, D.: Aggregate and Verifiably Encrypted Signatures from Multilinear Maps without Random Oracles. In: Park, J.H., Chen, H.-H., Atiquzzaman, M., Lee, C., Kim, T.-h., Yeo, S.-S. (eds.) ISA 2009. LNCS, vol. 5576, pp. 750-759. Springer, Heidelberg (2009)

RSA78. Rivest, R.L., Shamir, A., Adleman, L.M.: A method for obtaining digital signatures and public-key cryptosystems. Commun. ACM 21(2), 120-126 (1978)

RSA02. PKCS \#1: RSA Encryption Standard. Version 2.1. RSA Laboratories (June 2002), ftp://ftp.rsasecurity.com/pub/pkcs/pkcs-1/pkcs-1v2-1.pdf

Sco11. Michael Scott. MIRACL library (2011), http://www.shamus.ie/

Smi12. Smith, P.: BGP routing table analysis (2012), http://thyme.rand.apnic.net/. See historical data-e.g., APNIC analysis summary for (September 7, 2012), http://thyme.apnic.net/ap-data/2012/09/07/0400/mail-global

Sri12. Sriram, K. (ed.): BGPSEC Design Choices and Summary of Supporting Discussions. The Internet Engineering Task Force (IETF) Network Working Group (July 2012), http://tools.ietf .org/html/ draft-sriram-bgpsec-design-choices-02

SVS $^{+}$09. Sharmila Deva Selvi, S., Sree Vivek, S., Shriram, J., Kalaivani, S., Pandu Rangan, C.: Security analysis of aggregate signature and batch verification signature schemes. Technical Report 2009/290, Cryptology ePrint archive (2009), http://eprint.iacr.org

SVSR10. Sharmila Deva Selvi, S., Sree Vivek, S., Shriram, J., Pandu Rangan, C.: Identity based partial aggregate signature scheme without pairing. Report 2010/461, Cryptology ePrint archive (2010), http://eprint.iacr.org

Van92. Vanstone, S.: Responses to NIST's proposal. Communications of the ACM 35, 50-52 (1992)

WM08. Wen, Y., Ma, J.: An aggregate signature scheme with constant pairing operations. In: CSSE (3). IEEE Computer Society (2008)

XZF05. Xu, J., Zhang, Z., Feng, D.: ID-Based Aggregate Signatures from Bilinear Pairings. In: Desmedt, Y.G., Wang, H., Mu, Y., Li, Y. (eds.) CANS 2005. LNCS, vol. 3810, pp. 110-119. Springer, Heidelberg (2005)

YCK04. Yoon, H., Cheon, J.H., Kim, Y.: Batch Verifications with ID-Based Signatures. In: Park, C., Chee, S. (eds.) ICISC 2004. LNCS, vol. 3506, pp. 233-248. Springer, Heidelberg (2005)

ZSN05. Zhao, M., Smith, S.W., Nicol, D.M.: Aggregated path authentication for efficient BGP security. In: ACM Conference on Computer and Communications Security, pp. 128-138. ACM (2005) 